\title{
Estudio del pH y la concentración de glicerol para la producción de antígeno recombinante de Plasmodium vivax usando Pichia pastoris
}

\section{Study of $\mathrm{pH}$ and glycerol concentration to production of recombinant Plasmodium vivax antigen using Pichia pastoris}

\author{
Omar Pillaca-Pullo, Valker Feitosa, Irene Soares \& Adalberto Pessoa-Jr \\ Facultad de Ciencias Farmacéuticas, Universidad de São Paulo, Brasil
}

DOI: https://doi.org/10.33017/RevECIPeru2014.0009/

\section{Resumen}

La levadura metilotrófica Pichia pastoris es ampliamente usada como un sistema eucariota para expresar proteínas recombinantes. Más de 500 proteínas recombinantes fueron expresas por $P$. pastoris con niveles de expresión que alcanzan hasta el $80 \%$ de proteínas totales secretadas y hasta $30 \%$ de proteínas totales de la célula. El proceso productivo está compuesto por una fase de crecimiento con glicerol y luego por una fase de inducción con metanol. El control de las condiciones de cultura $(\mathrm{pH}$, temperatura, concentración de inductor, fuente de carbono y nitrógeno, etc.) son factores importantes tanto para el crecimiento de la levadura como para la producción de la proteína. Por su parte, la malaria es una enfermedad con gran importancia para la salud pública mundial debido a su alta prevalencia principalmente en los países tropicales. En los últimos años hubo un avance en la investigación de vacunas contra las especies de Plasmodium, dentro de las principales proteínas antigénicas están el Antígeno de Membrana Apical (AMA) y la Proteína de Superficie de Merozoíto (MSP) que pueden ser administradas aisladas, en combinación o conjugadas (quimera). En este estudio fueron evaluados el pH inicial del medio (3.0 a 8.0) y la concentración inicial de glicerol ( 0 a $\left.60 \mathrm{~g} . \mathrm{L}^{-1}\right)$ en el crecimiento la levadura, después de determinadas las cantidades de biomasa seca obtenida en cada punto, se tomaron tres valores de $\mathrm{pH}\left(6.0,7.0\right.$ y 8.0) y tres concentraciones diferentes de glicerol $\left(10,30\right.$ y $\left.50 \mathrm{~g} . \mathrm{L}^{-1}\right)$ y se realizaron experimentos con combinaciones de dichos parámetros. Los datos mostraron que la condición de mejor producción del antígeno recombinante de Plasmodium vivax (PvAMA166-MSP119) fue a pH 7.0 y $30 \mathrm{~g} \cdot \mathrm{L}^{-1}$ de glicerol y que altas concentraciones de glicerol provocan una mayor disminución del pH final afectando la producción del antígeno recombinante y aumentando el riesgo de proteólisis. Finalmente se recomienda evaluar dicha producción en biorreactor donde se lleve un control adecuado del pH con uso de hidróxido de amonio para corregir el pH el cual también es aprovechado por la levadura como fuente de nitrógeno.

Descriptores: Pichia pastoris, Plasmodium vivax, Antígeno recombinante

\begin{abstract}
The methylotrophic yeast Pichia pastoris is widely used as a eukaryotic system for expressing recombinant proteins. Over 500 recombinant proteins were expressed by $P$. pastoris with expression levels reaching up to $80 \%$ of total secreted proteins and up to $30 \%$ of total cell protein. The production process consists of a growth phase with glycerol and then an induction phase with methanol. Control culture conditions $(\mathrm{pH}$, temperature, concentration of inducer, carbon and nitrogen source, etc.) are important for the growth of yeast to the production of the protein factors. On the other hand, malaria is a disease of great importance to global public health because of it is high prevalence mainly in tropical countries. In recent years there has been a breakthrough in the research
\end{abstract}


of vaccines against Plasmodium species within the major antigenic proteins are the Antigen Membrane Apical (AMA) and Merozoite Protein Surface (MSP) that can be administered singly, in combination or conjugate (chimera). In this study were evaluated initial medium pH (3.0 to 8.0) and the initial concentration of glycerol (0 to $60 \mathrm{gL}^{-1}$ ) on growth of yeast, after analyzed amounts of dry biomass obtained at each point, were taken three $\mathrm{pH}$ values $\left(6.0,7.0\right.$ and 8.0) and three different concentrations of glycerol $\left(10,30\right.$ and $\left.50 \mathrm{gL}^{-1}\right)$ and experiments were performed with combinations of these parameters. The data showed that the best condition producing the recombinant antigen of Plasmodium vivax (PvAMA166-MSP119) was at $\mathrm{pH} 7.0$ and $30 \mathrm{gL}^{-1}$ of glycerol and that high concentrations of glycerol cause higher final $\mathrm{pH}$ decrease affecting the production of recombinant antigen and increasing the risk of proteolysis. Finally it is recommended to evaluate this production in bioreactor where adequate control of $\mathrm{pH}$ is carried with use of ammonium hydroxide to adjust the $\mathrm{pH}$ which is also used by the yeast as a nitrogen source.

\section{Keywords: Pichia pastoris, Plasmodium vivax, Recombinant antígen}

\section{Introducción}

En la década de los 70, la Phillips Petroleum en conjunto con el Salk Institute

Biotechnology/Industrial Associates, Inc. (SIBIA, La Jolla, CA) desarrollaron la Pichia pastoris como organismo para la producción de proteínas heterólogas, en la presencia de metanol, en cultivos de elevada densidad celular (>100 g.L-1). Los investigadores aislaron el promotor y el gen de la enzima alcohol oxidasa 1 (AOX1) y desarrollaron protocolos para la manipulación genética de la levadura [1].

Desde entonces, la $P$. pastoris, una levadura metilotrófica, o sea, capaz de metabolizar metanol, ha llegado a revelarse como una de las levaduras mas versátiles para la expresión de proteínas heterólogas [2]. Esta levadura es de particular interés debido: 1) A su promotor pAOX1 - promotor del gen que codifica para la enzima alcohol oxidasa 1 - inducido por metanol; 2) A la facilidad y simplicidad de manipulación genética, siendo ya conocidos y bien caracterizados diversos vectores de expresión, habiendo inclusive kits de expresión comerciales disponibles; 3) A su capacidad para liberar para el medio extracelular las proteínas heterólogas producidas; 4) A su capacidad para efectuar modificaciones pós-traduccionales incluyendo glicosilación, metilación, acilación e formación de ligaciones disulfuro; 5) A la posibilidad de operación en elevadas concentraciones celulares con elevados rendimientos de producción; 6) A la posibilidad de operación en medios de cultivo de costos reducidos y 7) al hecho de ser una levadura anaeróbica facultativa, posibilitando la operación en ambiente aeróbico, lo que disminuye los efectos negativos de algunos productos secundarios relativos a los procesos fermentativos, como el etanol [3].
Las condiciones de cultivo son importantes factores en el rendimiento de producción de las proteínas heterólogas en este sistema de expresión. La definición del medio de cultivo óptimo para el crecimiento y producción es una de las principales etapas en el desarrollo de un proceso de producción [4]. Las variables que presentan influencia directa en el rendimiento de expresión son el $\mathrm{pH}$, la temperatura y la concentración de oxigeno disuelto, además de la composición del medio, principalmente en lo que respecta a la fuente de carbono. En la literatura, los cultivos de $P$. pastoris presentan valores de $\mathrm{pH}$ que varían entre 3 y 7 , siendo que, fuera de este rango de valores, se verifican velocidades de crecimiento celular mas bajas, [38] puede ocurrir precipitación de sales del medio y la estabilidad de las proteínas puede ser afectada. Con respecto a la temperatura, $30^{\circ} \mathrm{C}$ es establecida como temperatura óptima de crecimiento [5].

Por otra parte, la malaria causada por protozoarios del género Plasmodium y transmitida entre seres humanos por hembras de mosquitos de anófeles, representa uno de los mayores desafíos en el control de la salud pública. Se estima que $40 \%$ de la población mundial vive en situación de riesgo de malária y de uno a dos millones morirán anualmente, cuya mayoría son niños [6] . Apesar de varios esfuerzos en el desarrollo de insecticidas y antimaláricos, la enfermedad aún no fue controlada. Esto se debe principalmente al aumento de la resistencia de los mosquitos a los insecticidas y del parásito a los fármacos [7], [8], [9], [10] y [11].

Actualmente,las investigaciones para desarrollar vacunas contra la malaria están direccionadas principalmente contra Plasmodium falciparum y $P$. vivax que son los principales causantes de la enfermedad. El desarrollo de una vacuna efectiva 
contra estos parásitos enfrenta dificultades impuestas por la complejidad del ciclo de vida, el extenso polimorfismo antigénico y la capacidad de evasión de la respuesta inmunológica. Además de eso, la falta de información de los principales mecanismos y moléculas parasitas que naturalmente inducen resistencia a la infección por malaria dificulta la selección de antígenos candidatos [6].

Los antígenos AMA-1 y MSP-1 son fuertes candidatos para desarrollar vacunas contra Plasmodium vivax, estas dos proteínas ya fueron utilizadas aisladamente [12], [13], en combinación [14] y mas recientemente está siendo estudiada la antigenicidad de estas proteínas conjugadas. Estudos en $P$. palciparum mostraron que la fusón de AMA-1 y MSP1-19 como una proteína conjugada no altero su estructura, soportando la utilización de proteínas quiméricas como potencial vacuna contra malaria [15].

El objetivo de nuestro trabajo fue estudiar en que medida la concentración de glicerol y el pH del medio influyen en la producción del antígeno recombinante de P. vivax (PvAMA166-MSP119).

\section{Materiales y métodos}

\subsection{Evaluación del pH y la concentración de Glicerol en el crecimiento}

El crecimiento de P. pastoris fue evaluado en frascos Erlenmeyer con deflectores de $250 \mathrm{~mL}$ conteniendo $50 \mathrm{~mL}$ de medio BMGY (Buffered Medium GlycerolYeast Extract), la evaluación del pH fue en el rango de 3.0 - 8.0 en medio de cultura con 10 g.L $\mathrm{L}^{-1}$ de glicerol y la concentración del glicerol fue evaluada en el rango de $0-60.0$ g. $\mathrm{L}^{-1}$ en medio de cultura a $\mathrm{pH}$ 6.0. Los frascos fueron cultivados a $30^{\circ} \mathrm{C}$ durante 24 horas en shaker New Brunswick Scientific ${ }^{\circledR}$, el medio fue centrifugado a $5000 \mathrm{rpm}$ durante 10 minutos y la biomasa fue colocada en estufa a $50{ }^{\circ} \mathrm{C}$ durante 48 horas para su cuantificación por peso seco.

\subsection{Producción de antígeno recombinante de $P$. vivax}

Después de la etapa de crecimiento, fue realizada la producción del antígeno mediante inducción con metanol al $1 \%$ cada 24 horas durante 48 horas. Se seleccionaron las condiciones de concentración de glicerol $(10.0,30.0$ y 50.0 g.L-1) y pH $(6.0,7.0$ y 8.0$)$ ya que mostraron mejor producción de biomasa.
Después de realizada la producción del antígeno, los cultivos fueron centrifugados a $5000 \mathrm{rpm}$ durante 10 minutos, la biomasa fue colocada en estufa para su cuantificación, por su parte el sobrenadante fue utilizado para la medir el $\mathrm{pH}$ y para diferenciar la producción del antígeno recombinante por electroforesis SDS-PAGE en cámara vertical BIORAD $^{\circledR}$.

\section{Resultados y discusión}

La cantidad de proteínas heterólogas producidas en $P$. pastoris, tiene una relación directa con la cantidad de biomasa producida durante la fase de crecimiento, a su vez la cantidad de biomasa depende de la cantidad de la fuente de carbono presente en el medio. En la Fig. 1 se muestran las cantidades de biomasa $\left(\mathrm{g} \cdot \mathrm{L}^{-1}\right)$ obtenidas después de 24 horas a diferentes concentraciones iniciales de glicerol. La biomasa producida sigue un comportamiento casi linear con el aumento de la concentración de glicerol hasta $50.0 \mathrm{~g} . \mathrm{L}^{-1}$; concentración que supera a la concentración de $40 \mathrm{~g} . \mathrm{L}^{-1}$ recomendada por Digan et al., 1989, donde hace mención a que cantidades mayores de glicerol son toxicas para la levadura disminuyendo la cantidad de biomasa producida, tal como ocurre a 60.0 g. $\mathrm{L}^{-1}$ de glicerol. [16]

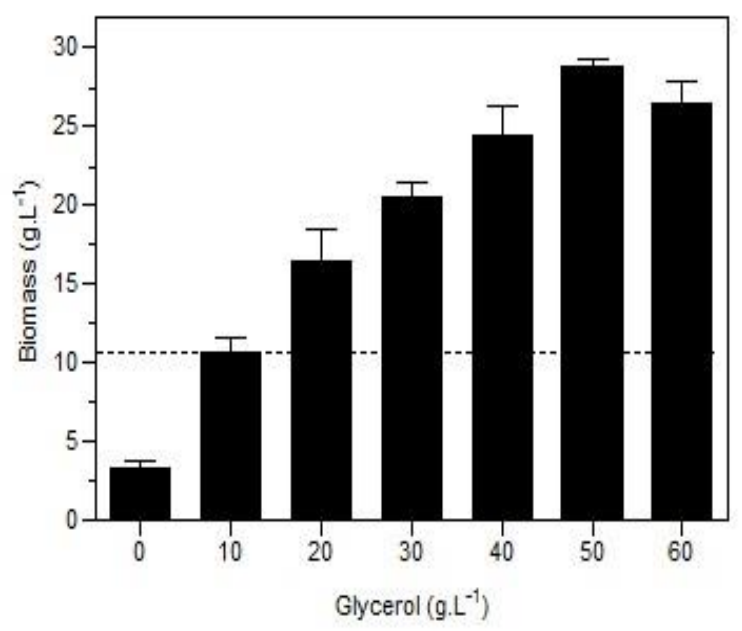

Figura 1: Producción de biomasa en diferentes concentraciones de glicerol. Se muestra la línea de referencia en condiciones estándar $(\mathrm{pH} 6.0$ y Glicerol $\left.10 \mathrm{~g} \cdot \mathrm{L}^{-1}\right)$.

Por su parte, como muestra la Fig. 2, la influencia del $\mathrm{pH}$ en la producción de biomasa no es tan notable, tal y como Cregg et al. 1993 mostraron que $P$. pastoris crece bien sobre un amplio rango de $\mathrm{pH}$, desde 3 
hasta 7 , con un mínimo efecto sobre la velocidad de crecimiento. [17]

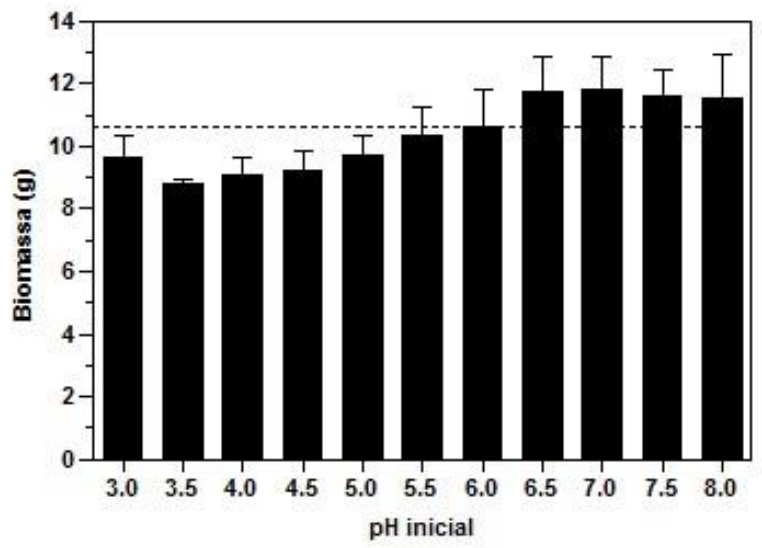

Figura 2: Producción de biomasa en diferentes valores de $\mathrm{pH}$ inicial. Se muestra la línea de referencia en condiciones estándar ( $\mathrm{pH} 6.0$ y Glicerol 10 g. $\mathrm{L}^{-1}$ ).

De los dos experimentos anteriores se realizó la selección de las concentraciones de glicerol a 10.0, 30.0 y 50.0 g.L-1, valores donde la producción de biomasa guarda relación directa con la concentración de glicerol; los valores de $\mathrm{pH}$ inicial de 6.0, 7.0 y 8.0, rango de $\mathrm{pH}$ donde se obtuvieron la mayor producción de biomasa. Estas condiciones fueron analizadas combinatoriamente para determinar aquella con la mejor producción del antígeno recombinante. De este experimento fue obtenido lo siguiente: El aumento de la concentración de glicerol genera un aumento en la cantidad de biomasa producida (Fig. 3) y una mayor disminución del pH al final del cultivo (Fig. 4). Del análisis realizado con electroforesis SDS-PAGE (Fig. 5), se obtuvo que la concentración de antígeno recombinante obtenida fue a pH 7.0 y $30.0 \mathrm{~g} \cdot \mathrm{L}^{-1}$ de glicerol, es decir la producción del antígeno es más influenciada por el $\mathrm{pH}$ del medio que por la cantidad de biomasa producida. La disminución de la producción a 50.0 g. $\mathrm{L}^{-1}$ es debido a que todo el glicerol no es consumido y este inhibe la expresión de las proteínas recombinantes, disminuyendo la productividad de la levadura [18], [19] (Boze et al. 2001; Files et al., 2001). Por otra parte la baja del pH aumenta la posibilidad de proteólisis del producto y tiene relación con la mayor actividad de las proteasas presentes en el medio [20], [21] (Wolf K. 1996; Inan et al. 1999). Para finalizar es necesario mencionar que la producción puede ser mejorada aún más a partir de uso de biorreactores donde se lleve un control del pH durante todo el tiempo de cultivo, se recomienda mantener el $\mathrm{pH}$ a través de la adición de hidróxido de amonio el cual también es utilizado por $P$. pastoris como fuente de nitrógeno.



Figura 3: Producción de biomasa en los experimentos con parámetros combinados $(\mathrm{pH} y$ Glicerol).

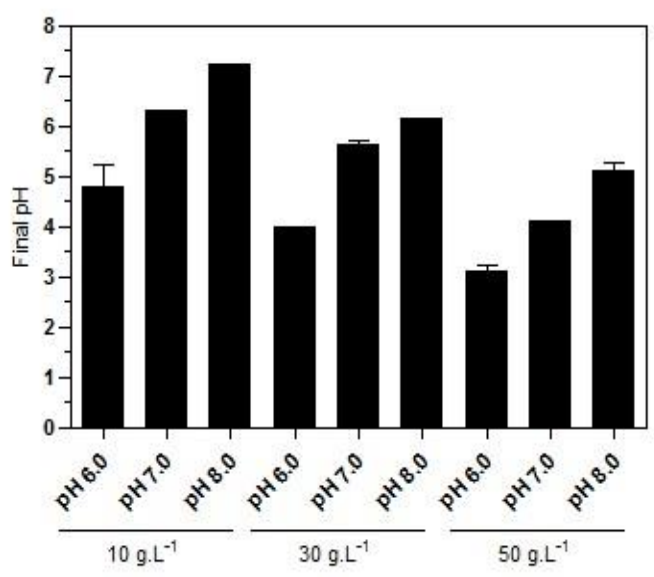

Figura 4: Valores de $\mathrm{pH}$ final en los experimentos con parámetros combinados ( $\mathrm{pH}$ y Glicerol).

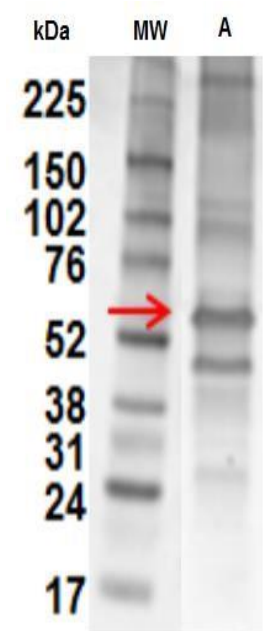

Figura 5: Gel SDS-PAGE mostrando la banda del antígeno recombinante (PvAMA166-MSP119 60 
$K D a)$ a la condición de mayor producción $p H 7.0$ y 30.0 g.L-1 de glicerol.

\section{Conclusiones}

La concentración de glicerol (10.0 - 50.0 g.L-1 $)$ presente en el medio guarda relación directa con la cantidad de biomasa producida, además altas concentraciones provocan una mayor disminución del $\mathrm{pH}$ final afectando la producción del antígeno recombinante y aumentando el riesgo de proteólisis. La mejor condición para producir antígeno recombinantes (PvAMA166-MSP119) en frascos agitados fue a pH 7.0 y 30.0 g. $\mathrm{L}^{-1}$ de glicerol.

\section{Agradecimientos}

Laboratorios de Biotecnología FarmacéuticaFacultad de Ciencias Farmacéuticas de Sao Paulo.

\section{CONCYTEC.}

\section{Referencias}

[1] J.L. Cereghino, J.M. Cregg, FEMS microbiology reviews. 24 (2000) pp 45- 66.

[2] A. Berlec, B. Strukelj. Journal of industrial microbiology \& biotechnology 91 (2013) pp 257-274.

[3] G. Potvin, A. Ahmad, Z. Zhang 64 (2012) pp 91-105.

[4] R. Daly, M. T. W. Hearn, Journal of molecular recognition. 18 (2005) pp 11938.

[5] O. Cos, R. Ramón, J.L. Montesinos, F. Valero, Microbial cell factories. 5 (2006) pp 17.

[6] M. H. Rodríguez-López, Rev Biomed. 19 (2008) pp 61-79.

[7] F. Collins, N. Besansky. Science. 264 (1994) pp 1874-1875.

[8] M.J. Mackinnon, I.M. Hastings. Trans Roy Soc Trop Med HYg. 92 (1998) 188195.

[9] J. Hemingway, Parassitologia. 41 (1999) pp 315-318

[10] R.S. Phillips. Clin Microbiol Rev 14 (2001) pp 208-226.

[11] P. Wilairatana, S. Krudsood, S. Treepraswrtsuk, K. Chalemrut, S. Looareesuwan. Arch Med Res. 33 (2002) pp 416-421.
[12] F. Gentil , D.Y. Bargieri, J.A. Leite , K.S. Françoso, M.B. Patricio. Vaccine 28 (2010) pp 6183-6190

[13] L. Lacerda, R. Toshio, I. Silva, E. Martins. Vaccine 26 (2008) pp 12041213

[14] E. Malkin, J. Hu, Li Z, Z. Chen, X. Bi. Vaccine, 26 (2008) pp 6864-6873.

[15] H. Peng, Y. Hu, A. Zhou, C. Jin, W. Pan. Malaria Journal, 9 (2010) pp 76 [16] M. E. Digan, S. V. Lair, R. A. Brierley, R. S. Siegel. Bio/Technology 7 (1989) pp 160164.

[17] J.M. Cregg, T.S. Vedvick, and W.C. Raschke. Bio/Technology, 11 (1993) pp 905-910

[18] H. Boze, L. Celine, Ch. Patrick, R. Fabien. Process Biochemistry, 36 (2001) pp 907-913.

[19] D. A Files, M. Ogawa, C.H. Scaman and S.A. Baldwin. Enzyme and Microbiological Technology, 26 (2001) pp 335-340.

[20] K. Wolf, Springer Verlag Berlin Heildelberg, (1996) pp 203-253.

[21] M. Inan, V. Chiruvolu, K.M. Eskridge, G.P. Vlasuk, K. Dickerson, Enzyme Microb. Technol., 24 (1999) pp 438. 